\title{
Landschaftsplanung auf Gemeindeebene am Beispiel Oberwil, Kanton Baselland
}

\section{Einleitung}

«Es ist nun wirklich an der Zeit, daß wir uns auf unsere Bedürfnisse besinnen, meine Damen und Herren,» ruft ein sehr engagierter Bürger in den Gemeindesaal. Dies während der Diskussion an einer der Orientierungsversammlungen über die Landschaftsplanung Oberwil. Und fährt weiter: "Ich glaube nicht, daß ich kleinlich bin: Ich habe mich stets für das regionale Wohl eingesetzt. Aber jetzt geht es um unseren Landschaftsraum, um die Existenzgrundlage unserer Bauern. Jetzt werden die Weichen gestellt hinsichtlich dessen, was mit unseren Feldern geschieht. Wird darauf weiterhin das Vieh weiden, das Getreide wogen, im Herbst das Muttfeuer brennen, werden wir auch künftig frei durch die Obstkulturen wandern und den unversehrten Ausblick auf die Jurakette genießen? Oder lassen wir es zu, daß sukzessive große Komplexe unserer freien Landschaft eingehagt werden, damit dort die Mitarbeiter der X-Y-Versicherung oder der Z-Bank ihren Freizeitaktivitäten nachgehen können? Gewiß, auch die Leute aus der Stadt haben ein echtes Bedürfnis nach Erholung, das sie in den engen Grenzen des Stadtkantons kaum befriedigen können. Aber - muß da ausgerechnet auf unsere Gemeinde ausgewichen werden? Haben wir denn nicht schon genug für das regionale Wohl getan? Ich denke dabei nicht nur an die Depotanlagen der Baselland Transport oder an das Gymnasium, das als störender, häßlicher Baukomplex mitten in die Landwirtschaft verlegt wurde, weil sich dort der Kanton den Boden billig zu verschaffen wußte. Wir haben darüber hinaus zwei große, von Auswärtigen stark genutzte Wochenendhausgebiete, Glashausgärtnereien, Familiengartenareale, das ausgedehnte Lehmabbaugebiet für die Ziegelei und nicht zuletzt Hundesport- und Reitanlagen. - Die Reiterei, ein Kapitel für sich! Ich reite selber gerne. Aber wenn ich an unsere übernutzten Wälder denke, komme ich nicht darum herum festzustellen, daß das Reiten, das ja nicht nur von unserem Betrieb ausgeht, sondern auch von den zahlreichen andern unserer Nachbargemeinden, ein ernsthaftes Problem darstellt.

Mit dem Landschaftszonenplan, der uns hier zur Beschlußfassung vorgelegt wird, haben wir es in der
Hand, in die Vielfalt der sich zum Teil widersprechenden Interessen Ordnung zu bringen. Es geht darum, sie in ein vertretbares, ausgewogenes Verhältnis zu bringen.»

Das Votum richtet sich also nicht gegen die entwicklungsbedingte Umstrukturierung des Landschaftsraumes. Wie sich im weiteren Verlauf der Debatte herausstellt, hat der Votant - und mit ihm eine große Zahl weiterer Einwohner - die sogenannte «Zone für Intensiverholung» im Visier, eine Planungsmaßnahme, auf die noch einzugehen ist. Auslöser für diese Veranstaltung war die Gemeindeversammlung vom 24. Juni 1976, welche mit groBem Mehr gegen 2 Stimmen die Revision der Ortsplanung beschlossen hatte. Der Auftrag umfaßte neben der Revision der Bauzonenvorschriften 1966 auch die Ausarbeitung eines kommunalen Landschaftszonenplanes mit zugehörigen Vorschriften. Darüber sei im folgenden berichtet.

\section{Allgemeines}

\section{Landschaftsplanung}

Ein Begriff, der nicht genau aussagt, was er meint und doch von jedermann anscheinend richtig verstanden wird. Für mich ist das ein kleines Phänomen. Ich bin mich als Planer gewohnt, um Worte zu ringen, die im Bereich der Planung Tatbestände erklären, so daß sie genau das aussagen, was gemeint ist. Und ich bin mich ebenso gewohnt festzustellen, daß diese abgewogenen Worte mißverstanden werden, daß jeder, der sie aufnimmt, seine persönliche Interpretation hineinlegt, die bisweilen sogar dem direkt entgegensteht, was die gebotene Erklärung festhält. So rankt sich dann ein Bündel von individuellen Vorstellungen um die gemachte, eigentliche Aussage. Anders also beim Begriff «Landschaftsplanung». Vielleicht ist es gerade die sachlich verschwommene Bezeichnung, welche die allgemeine Meinung darauf einigt, was gemeint ist.

Marc Bütler, dipl. Arch. BSA, Planer BSP, St. Jakobsstraße 2 , 4132 Muttenz. 
Von der Sache her ist klar, daß es nicht darum geht, einen Plan zu machen, dessen Verwirklichung eine Landschaft entstehen läßt, wie etwa beim Planen und Bauen eines Hauses, eines Quartiers oder einer Straße. Die Landschaft ist ja längst geschaffen. Aber, sie wandelt sich stetig unter Druck der Bedürfnisse, die auf ihrem Boden nach Verwirklichung drängen.

Eindeutiger als bei der Siedlungsplanung, der Verkehrsplanung oder der Planung öffentlicher Einrichtungen ist die Landschaftsplanung darauf gerichtet, die an den Raum gestellten Nutzungsforderungen zu ordnen und gegebenenfalls zu beschränken. Wir werden im Detail noch darauf zurückkommen. Das planmäßige Vorgehen ist es, was dabei im Wort Landschaftsplanung zum Ausdruck kommt.

\section{Aufgabe der Landschaftsplanung}

Im Zentrum des Interesses stehen stets das Bedürfnis nach Landschaftsschutz und die Frage nach dem Maß und der Zulässigkeit von Nutzungsformen, die nicht der Agrarnutzung im engeren Sinne zuzuordnen sind. In unserem Beispiel ist, zufolge der Stadtnähe, eine besonders deutliche Nachfrage nach Erholungseinrichtungen und Freizeitanlagen zu verzeichnen.

Ohne ordnenden Plan vollzieht sich die Veränderung der überkommenden Substanz dabei rein zufällig. Die kantonale Baugesetzgebung fordert primär eine konsequente Abgrenzung zwischen Baugebiet einerseits und Land- und Forstwirtschaftsgebiet anderseits. Im Falle des Kantons Baselland ist festgelegt, daß das letztere der land- und forstwirtschaftlichen Nutzung vorzubehalten sei, wobei der Regierungsrat Ausnahmen für andere Nutzungen gewähren kann, die sich im Baugebiet als nicht tragbar erweisen. Den Gemeinden steht indessen $\mathrm{zu}$, für spezielle Bedürfnisse entsprechende Zonen im ganzen Gemeindebann auszuscheiden. Solange nun eine Gemeinde von diesem Angebot keinen Gebrauch macht, ist es demnach in Wirklichkeit so, daß der Regierungsrat von Fall zu Fall direkt in die Gestaltung des Freiraumes eingreift. Das ist für das Gemeinwesen eine wenig vorteilhafte Situation. Gerade die Oberwiler haben das am eingangs erwähnten Reitproblem deutlich erfahren: Als nämlich die Absicht bekannt wurde, einen weiteren Reitbetrieb in der Gemeinde zu erstellen, beauftragte die Gemeindeversammlung in einer Resolution den Gemeinderat, dieses Vorhaben zu verhindern. Das Verwaltungsgericht stellte dazu fest, daß es nicht angehe, ohne Erlaß von kommunalen Landschaftszonen-Vorschriften bestimmte Nutzungen auszuschließen. Die bundesgerichtliche Rechtssprechung hat darüber hinaus wiederholt klargestellt, daß berechtigte Nutzungsansprüche durch
Planungsmaßnahmen nicht unterbunden, wohl aber an dafür geeignete Standorte verwiesen werden können.

Als taugliches Instrument hiefür bietet sich der Zonenplan an, welcher sich über längere Zeit nur mit dem Baugebiet ordnend auseinandersetzte und erst in den letzten Jahren auch das übrige Gemeindegebiet mit einzubeziehen begann.

\section{Landschaftsnutzung - Landschaftsschutz}

Landschaftsplanung ist also konfrontiert mit den Forderungen nach Landschaftsschutz sowie nach Gewährleistung berechtigter Landschaftsnutzung an dafür geeigneter Lage. Dabei stellt sich ein enges Abhängigkeitsverhältnis von Schutz und Nutzung heraus; denn Landschaftsschutz bedeutet stets das Unterordnen von Nutzungsansprüchen unter ein bestimmtes Schutzziel: Ein wertvolles Landschaftselement, das sich beispielsweise durch besondere Naturbezogenheit, Vegetationsvielfalt und spannungsvollen Wechsel in der Wald-Flurverteilung auszeichnet, läßt sich nur dadurch schützen, daß diesem Charakter wesensfremde Nutzungsformen, wie etwa Glashausgärtnereien oder Aufforstungen, in seinem Bereich konkret ausgeschlossen werden. Dabei ist sogleich darauf hinzuweisen, daß Landschaftsschutzmaßnahmen nicht allein ästhetischvisuelle Kriterien berücksichtigen dürfen, sondern ökologische ebenso mit einzubeziehen haben. Am Beispiel: Der besondere Schutz von Flora und Fauna eines von der Erscheinung her nicht besonders wertvollen Waldsaumes kann sich etwa deshalb aufdrängen, weil die angrenzende Agrarfläche aus Gründen einer rationellen Bewirtschaftung vom ökologisch wichtigen Baumbestand völlig ausgeräumt wurde. Die Schutzmaßnahme hat in diesem Fall Ersatzfunktion.

«Unterordnen der im Landschaftsraum auftretenden Nutzungsformen unter eindeutig definierte Schutzziele» - das heißt konkret, daß, je nach Schwere des Konfliktes zwischen Nutzung und Schutzwürdigkeit des Raumes, die Nutzungsausübung wo nötig mittels $A$ uflagen eingeschränkt oder in bestimmten Fällen ausgeschlossen werden muß. Solche Auflagen sind durch Vorschriften des kantonalen Baurechtes gegeben, oder müssen auf dem Wege von Verordnungen, Reglementen oder von Vereinbarungen mit dem Nutzungsträger festgelegt werden.

\section{Technisches Vorgehen}

Um für die praktische Handhabung der Auflagen eine klare Grundlage zu schaffen, ist es zunächst nötig, einerseits den Landschaftsraum bestimmten Schutzkategorien mit konkreten Schutzzielen zuzuordnen und anderseits festzulegen, welche Nutzun- 


\begin{tabular}{|c|c|c|c|}
\hline $\begin{array}{l}\text { Allgemeines } \\
\text { Schutzziel }\end{array}$ & \multicolumn{3}{|c|}{$\begin{array}{l}\text { Planmäßiges Bewahren und Pflegen des Naturpotentiales zur langfristigen Sicherung von } \\
\text { natürlichen Produktionsgrundlagen, Naturschätzen und Erholungsräumen zum Wohle } \\
\text { der Allgemeinheit. } \\
\text { Schutz des Naturpotentiales aus ökologischen, ästhetischen und sozial-kulturellen Gründen. }\end{array}$} \\
\hline Schutzkategorie & Landschaftsschonung & Landschaftsschutz & Naturschutz \\
\hline $\begin{array}{l}\text { Spezielles } \\
\text { Schutzziel }\end{array}$ & $\begin{array}{l}\text { gemäß allgemeinem Schutz- } \\
\text { ziel, zusätzlich: } \\
\text { Bewahrung der Geschlossen- } \\
\text { heit eines zusammenhän- } \\
\text { genden Landschaftsraumes } \\
\text { zugunsten seiner langfristig } \\
\text { ökonomischen Nutzung. } \\
\text { Bewahrung von Landschafts- } \\
\text { einheiten und von land- } \\
\text { schaftsgerechten Nutzungs- } \\
\text { formen im Hinblick auf die } \\
\text { sozial-kulturelle Bedeutung } \\
\text { der Kulturlandschaft. }\end{array}$ & $\begin{array}{l}\text { gemäß allgemeinem Schutz- } \\
\text { ziel, zusätzlich: } \\
\text { Bewahrung typischer Land- } \\
\text { schaftsbilder. } \\
\text { Bewahrung und Pflege viel- } \\
\text { gestaltiger Kulturland- } \\
\text { schaften und ihrer natur- } \\
\text { betonten Bestandteile } \\
\text { Bewahrung ihrer kleinräu- } \\
\text { migen Gliederung und der } \\
\text { Wald-Flur-Verteilung. }\end{array}$ & $\begin{array}{l}\text { gemäß allgemeinem Schutz- } \\
\text { ziel, zusätzlich: } \\
\text { Bewahrung und Schutz aus } \\
\text { wissenschaftlichen Gründen. } \\
\text { Bewahrung und Pflege von } \\
\text { Landschaftsteilen und ihren } \\
\text { naturräumlichen Ausstat- } \\
\text { tungen von naturkundlichem } \\
\text { Interesse. } \\
\text { Bewahrung des Fortbestandes } \\
\text { schutzwürdiger Pflanzen- und } \\
\text { Tierarten und ihrer Lebens- } \\
\text { räume. }\end{array}$ \\
\hline $\begin{array}{l}\text { Räumlicher } \\
\text { Bezug }\end{array}$ & $\begin{array}{l}\text { Gesamte Fläche abzüglich } \\
\text { Baugebiete und Flächen der } \\
\text { höheren Schutzkategorien. }\end{array}$ & $\begin{array}{l}\text { Großräumige Gebiete } \\
\text { Flächen } \\
\text { Objekte } \\
\text { Umgebung von Natur- und } \\
\text { Kultur-Objekten. }\end{array}$ & $\begin{array}{l}\text { Flächen } \\
\text { Objekte }\end{array}$ \\
\hline
\end{tabular}

gen in diesen Schutzkategorien mit Auflagen oder Ausschluß zu belegen sind.

Mit Vorteil geschieht diese Arbeit auf übergeordneter Ebene und für größere, zusammenhängende Räume, beispielsweise für eine Region oder einen Kanton. Im Grunde ist nicht einzusehen, warum zumindest die einheitliche Definition der Schutzkategorien nicht sogar ganze Landesteile mit ähnlicher Landschaftsstruktur betreffen könnte. Dieser Überlegung folgend, haben es die Kantone Aargau, Baselland, Baselstadt, Bern und Solothurn unternommen, im sogenannten "Landschaftskonzept Nordwestschweiz» drei Schutzkategorien zu definieren, nämlich die Begriffe "Landschaftsschonung», "Landschaftsschutz» und "Naturschutz». Tabelle 1, welche aus dem zitierten Dokument entnommen ist, gibt diese Definitionen wieder (Regionalkonferenz der Regierungen der Nordwestschweiz, 1978). Das generelle Landschaftskonzept $\mathrm{NWCH}$ enthält zur Vertiefung der Definitionen überdies eine sogenannte "Verträglichkeitsmatrix», aus der hervorgeht, welche Nutzungsformen in den Schutzkategorien "Landschaftsschonung" und «Landschaftsschutz» mit Auflagen zu versehen oder auszuschließen sind. Die Kategorie «Naturschutz» ist in die Matrix nicht einbezogen, weil hier höchstens sehr extensive Nutzungen zugelassen werden können, die von Fall zu Fall festgelegt werden müssen.

Gemäß Vereinbarung unter den fünf Kantonen stützen sich die Planungsorgane dieser Region in ihrer technischen Arbeit auf das Landschaftskonzept NWCH als Arbeitsgrundlage ab. So hat beispielsweise die Regionalplanungsgruppe LaufentalThierstein-Dorneck bei der Ausarbeitung ihres Landschafts-Richtplanes die genannte Verträglichkeitsmatrix, auf die Gegebenheiten dieser Region abgestimmt, übernommen. Ich betrachte die eindeutige Festlegung des Verhältnisses zwischen Schutz und Nutzung, welche darin zum Ausdruck kommt, als eine zentrale Aufgabe der Landschaftsplanung, weshalb die Wiedergabe der Matrix hier angebracht erscheint. (Tabelle 2, Auszug aus dem technischen Bericht zum regionalen Richtplan Landschaft. Planergemeinschaft Regionalplanung Laufental-Thierstein-Dorneck, Oktober 1978).

\section{Landschaftsplanung auf übergeordneter Ebene}

Obschon grundeigentumsverbindliche Maßnahmen, wie Zonenausscheidung, Schutz- und Nutzungsvorschriften, im allgemeinen auf kommuna- 


\begin{tabular}{c|c|l}
\hline Schutz & & \\
Kategorie a & Kategorie b & Nutzung durch Urproduktion \\
\hline+ & + & Ackerbau und Graswirtschaft \\
$\triangleright$ & + & Intensivwirtschaft $\Leftrightarrow$ Erläuterungen) \\
$\triangleright$ & + & Intensivkulturen für Obst, Beeren, Rebbau \\
$\triangleright$ & $\triangleright$ & Meliorationsmaßnahmen \\
$\triangleright$ & + & direkt standortabhängige Bauten und Anlagen $\Leftrightarrow$ Erläuterung $)$ \\
- & $\triangleright$ & indirekt standortabhängige Bauten und Anlagen $(\triangle$ Erläuterung $)$ \\
+ & + & Forstwirtschaft \\
$\triangleright$ & + & Bestandesumwandlungen $(\Rightarrow$ Erläuterung) \\
$\triangleright$ & + & Rodungen und Aufforstungen \\
\hline
\end{tabular}

Kategorie a $=$ Landschaftsschutz

Kategorie $\mathrm{b}=$ Landschaftsschonung
+ Nutzung zulässig ohne besondere Auflagen

$\triangleright$ Nutzung zulässig mit Auflagen

- Nutzung nicht zulässig

\section{Erläuterung der Begriffe:}

Intensivwirtschaft ist bei intensiv genutzten Flächen gegeben, denen eine kleinräumliche Gliederung fehlt und deren Naturpotential durch starke Nutzung gefährdet ist.

Direkt standortabhängige Bauten und Anlagen sind Einrichtungen, deren Standort direkt von der Urproduktion abhängig ist. Beispiel: Aussiedlerhof.

ler Ebene zu erlassen sind, erweist es sich als notwendig, auf den übergeordneten Ebenen - Region, Kanton - ebenfalls eingehende Planungsarbeit zu leisten. Sie richtet sich vorwiegend auf Koordination und Vereinheitlichung der Planungsmaßnahmen auf kommunaler Stufe, wie am Beispiel «Landschaftskonzept NWCH» deutlich wurde, und findet ihren Niederschlag zumeist in Konzepten und Richtplänen. Es liegt dabei auf der Hand, daß diese Instrumente für die Landschaftsplanung besonders wichtig sind, weil ja der Landschaftsraum an der Gemeindegrenze nicht Halt macht und deshalb für einen nahtlosen Übergang der Zonenausscheidung gesorgt werden muß.

Für unser Fallbeispiel, die Landschaftsplanung Oberwil, waren zwei übergeordnete Planungsinstrumente maßgebend: das erwähnte Landschaftskonzept NWCH und der Regionalplan Landschaft des Kantons Baselland. Ersteres umfaßt neben den zitierten Begriffsdefinitionen einen provisorischen Richtplan im Maßstab 1:75000 (mit Gebietsausscheidungen bezüglich der Prioritäten Landschaftsschonung und Landschaftsschutz), Hinweise zur Landschaftspflege an Fallbeispielen, sowie die wichtigsten Ergebnisse der Bestandesaufnahme, auf welchen das Konzept fußt. Der Regionalplan Landschaft BL enthält eine sehr detailliert ausgearbeitete Disposition von Schutzgebieten, Erholungsgebieten und Einzelelementen. Er wird begleitet von einem umfassenden Erläuterungsbericht des
Indirekt standortabhängige Bauten und Anlagen sind Einrichtungen, deren Standort nur indirekt von der Urproduktion abhängig ist. Beispiele: Viehmästerei, Kleintierfarm, Gärtnerei, Grastrocknungsanlage, Familiengartenareal.

Bestandesumwandlung bedeutet ertragsorientierte Änderung eines naturnahen forstlichen Bestandes. Beispiel: Umwandlung von Laub- in Fichtenwald.

Regierungsrates an den Landrat, welcher gegenwärtig der parlamentarischen Beratung harrt (Regierungsrat des Kantons Basel-Landschaft, 3. Mai 1977).

\section{Landschaftsplanung am Beispiel Oberwil}

\section{Steckbrief der Gemeinde}

Oberwil mit 8000 Einwohnern ist eine Vorortsgemeinde der Stadt Basel, im Zentrum des zum Kanton Baselland gehörigen Leimentales gelegen. Das Baugebiet erstreckt sich über die Talmulde und die sie begleitenden Hänge des Bruderholzes im Osten und des Ausläufers des sundgauischen Hügellandes im Westen. Diese Lage bietet einerseits große Vorteile für eine enge Verknüpfung von Besiedlung und Naherholung, ist aber andererseits besonders heikel hinsichtlich Einpassung des Siedlungsbildes in die freie Landschaft.

Zum Ärger der Nachbarn bezeichnet sich Oberwil bisweilen als «Metropole des Leimentales». Im Hinblick auf seine Vielfalt des Dienstleistungsangebotes ist dieser eher scherzhaft gemeinten Charakterisierung eine gewisse Berechtigung nicht abzusprechen. Nicht zuletzt ausgelöst durch die Ortsplanungsrevision und die Absicht eines großen Detailhandelsunternehmens, in Oberwil ein regionales Einkaufszentrum zu errichten, ist indessen unter 


\begin{tabular}{l|c|l}
\hline Schutz & & \\
Kategorie a & Kategorie b & Nutzung durch Erholungseinrichtungen \\
\hline & & Einrichtungen ohne Landschaftsbelastung \\
+ & + & Wanderwege, Lehrpfade \\
+ & + & Reitwege \\
+ & + & Fitneßbahnen, z. B. Vita-Parcours \\
\hline & & Einrichtungen mit leichter Landschaftsbelastung \\
$\triangleright$ & + & Verkehrsunabhängige Rastplätze mit Ausstattungen, wie Feuerstellen, Picknick- \\
& & flächen, Schießanlagen \\
$\triangleright$ & + & Skipisten \\
$\triangleright$ & $\triangleright$ & Skiliftanlagen \\
$\triangleright$ & + & Zweckbauten, wie Unterstände, Kioske \\
- & $\triangleright$ & Landwirtschaftliche Freizeitanlagen (Geräteschuppen, Ställe) und Garten- \\
& $\triangleright$ & häuschen \\
$\triangleright$ & $\triangleright$ & Weiheranlagen für Fischzucht und Erholung \\
$\triangleright$ & $\triangleright$ & Wildpark, Tiergehege \\
$\triangleright$ & $\triangleright$ & Verkehrsabhängige Rastplätze in allen Ausbaustufen, mit Parkplätzen \\
$\triangleright$ & $\triangleright$ & Aussichtstürme \\
\hline & $\triangleright$ & Einrichtungen mit starker Landschaftsbelastung \\
\hline & $\triangleright$ & Erholungsschwerpunkte mit besonderen Bauten und Anlagen \\
- & $\triangleright$ & Schießanlagen \\
- & $\triangleright$ & Zonen für lärmverursachende Sportarten, z. B. Flugsport, Motorsport \\
& $\triangleright$ & Zonen für Intensiverholung, z. B. Sportstadien, Frei- und Hallenbäder, \\
& Kunsteisbahnen \\
\hline & & Zone für Camping und Wohnwagen \\
& & Zone für Feriensiedlungen und Wochenendhäuser \\
\hline
\end{tabular}

den Einwohnern der Gemeinde selber ein Prozeß der Neubesinnung in Gang gekommen. Vor allem die starke Verkehrsbelastung des Straßennetzes läßt bei vielen Ortsansässigen den Wunsch aufkommen, mehr «unter sich» zu sein und auf Hegemonieansprüche zu verzichten. Ein Großteil der Einwohner arbeitet in der nahen Stadt und hat seinerzeit den Wohnsitz nach Oberwil verlegt, um auf dem Land zu sein. Gerade deshalb wird der starke Strukturwandel der Landschaft als bedrohlich empfunden.

\section{Bürgerinitiative «Grünbuch»}

Das Bewußtsein einer Bedrohung des engeren Lebensraumes ist natürlich eine besondere Motivation für die Durchführung einer kommunalen Landschaftsplanung. Schon vor der diesbezüglichen Beschlußfassung durch die Gemeindeversammlung haben sich engagierte Einwohner ans Werk gemacht und in einem sogenannten "Grünbuch» den Mitbürgern die Dringlichkeit des Landschafts- schutzes vor Augen zu führen versucht. Das «Grünbuch» zeigt anhand vieler Fotos die Schönheiten der Landschaft, aber auch ihre fortschreitende Zerstörung auf und gibt eine Fülle von Anregungen zur Verbesserung der Verhältnisse, namentlich im Siedlungsbereich. Die Initiative hat großes Echo in der Bevölkerung gefunden und das Verständnis für die eigentliche Planungsarbeit wesentlich gefördert.

\section{Probleme im Oberwiler Landschaftsraum}

Eine sorgfältige Aufnahme des Ist-Zustandes und deren Auswertung hat zu folgendem Befund geführt:

Bereich Landwirtschaft:

Rückgang der hauptberuflichen Landwirtschaftsbetriebe von 22 im Jahre 1965 auf 13 in naher Zukunft. Besonders betroffen sind Betriebe im Ortskern. Bei Aufgabe aller existenzschwachen Höfe 


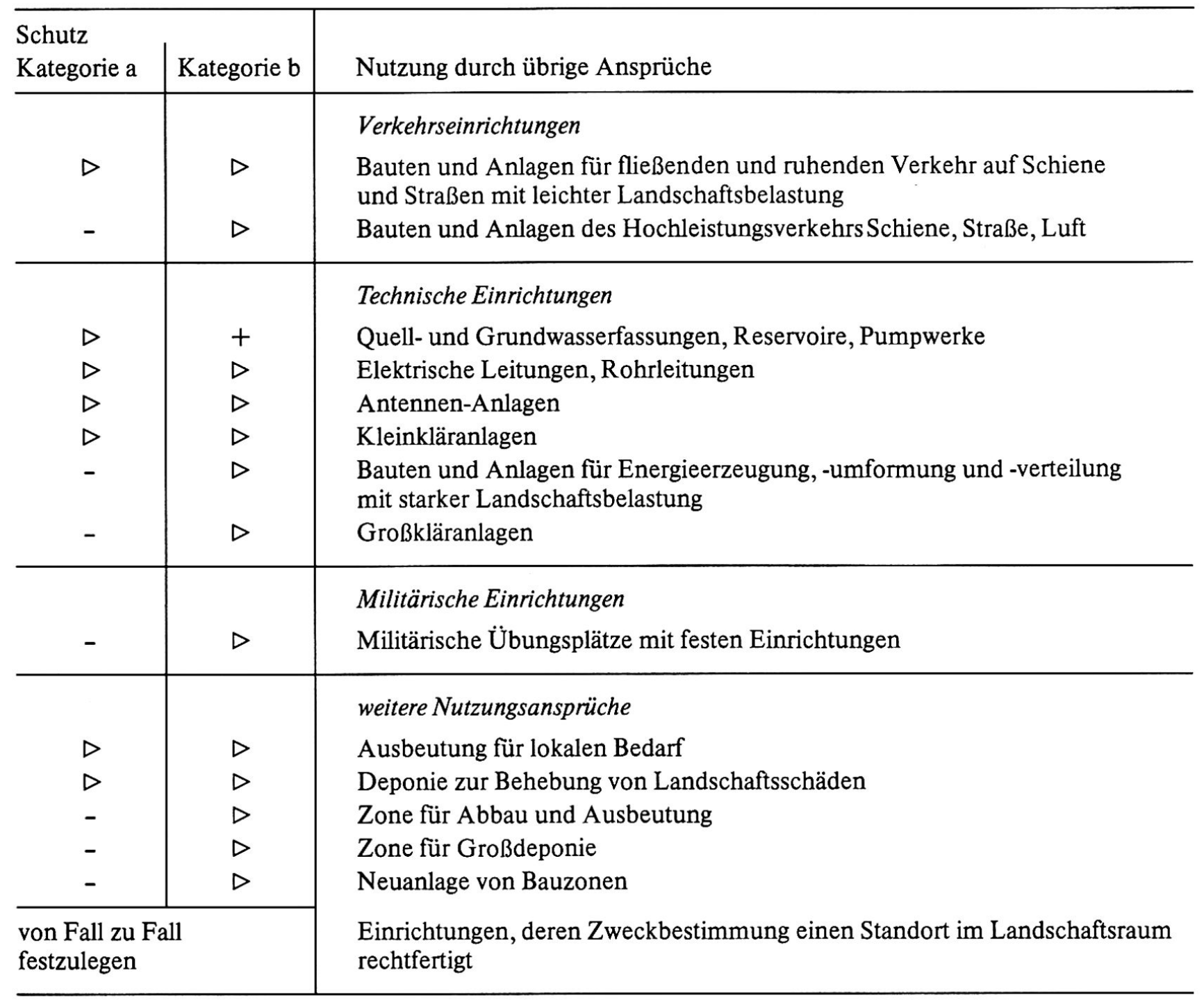

werden ca. 60 ha bester landwirtschaftlicher Nutzfläche frei, davon 24 ha im Baugebiet. Erschwerung einer wirtschaftlichen Produktivität durch ungünstige Parzellierung und hohen Grundeigentumsanteil von Nichtlandwirten in manchen Teilgebieten. Gefährdung der Kulturlandschafts-Substanz von vorrangiger Qualität durch: Glashausgärtnereien, zu groß angelegte Bauzonen, Sportanlagen, Einrichtungen der Intensiverholung, letztere besonders bei Eintreten auf die Intentionen des Regionalplanes.

Bereich Forstwirtschaft:

Anteil Privatwaldungen an Gesamtwaldfläche ca. $50 \%$ ! Ein großer Teil davon nicht mehr im Eigentum von aktiven Landwirten. Sehr starke Parzellensplitterung. Waldbestand meist überaltert, ertragsarm und von oft ungepflegter Erscheinung zufolge unsachgemäßer Bewirtschaftung in früheren Jahren. Bedrohung des landschaftstypischen Laubmischwaldes auf Privatparzellen durch Auffor- stung mit ortsfremden Holzarten. Beeinträchtigung des Waldjungwuchses, der natürlichen Bodenvegetation und teilweise der Tierwelt durch verschiedene Formen der Erholungsnutzung. Verschiedene Kleingehölze als wichtige Grünelemente im Baugebiet, sowie Feldgehölze in ihrem Fortbestand bedroht und ihre geordnete Pflege nicht gewährleistet.

Bereich Erholungsnutzung:

Gegenseitige Beeinträchtigung der verschiedenen Erholungsaktivitäten (Spazieren, Wandern, Waldlaufen, Reiten, Velofahren, Modellflugsport usw.) als typische Folge hoher Bevölkerungskonzentration. Lärmimmissionen. Teilweise Beeinträchtigung des Naturhaushaltes, Gefährdung der Kulturen durch motorisierte Picnicer. Im Verhältnis zur Bedeutung des Gebietes für die Naherholung ungenügende Steuerung der Erholungsaktivitäten und mangelnde Ausstattung mit zweckmäßigen, einfachen Einrichtungen. 
Andere Nutzungen:

Eingriffe in das natürliche Landschaftsgefüge durch bestehende oder geplante Anlagen wie: Ziegelei, Gymnasium, Birsigüberquerung Langmattstraße, Anschlußbauwerk an T 18A (Talentlastungsstraße).

Naturschutz und Landschaftspflege:

Beeinträchtigung der biologischen Vielfalt im Landschaftsraum unter anderem zufolge Ausräumung der natürlichen Elemente in einigen Teilen des landwirtschaftlichen Vorranggebietes. (Anderseits sticht Oberwil durch hohen, biologisch bewirtșchafteten Flächenanteil hervor.) Teilweise Vernachlässigung und dadurch Gefährdung der dichten, reich gegliederten Waldränder, Feld- und Ufergehölze, namentlich längs des Birsig.

\section{Lösungsrichtlinien}

In einem Zwischenbericht zur Bestandesaufnahme wurde eine Reihe von Lösungsrichtlinien zu den oben registrierten Problemen aufgelistet, deren wichtigste nachfolgend wiedergegeben werden:

- Schutz der landwirtschaftlichen Vorrangflächen vor Zweckentfremdung mittels Zonenvorschriften

- indirekt standortabhängige Bauten (Großgärtnereien, reine Mastbetriebe usw.) nur in Restgebieten oder außerhalb der Vorrangflächen im Tal zulassen (mittels Zonenvorschriften)

- Anlagen der Intensiverholung nur auf den heutigen Bedarf ausgerichtet entweder im Baugebiet und/oder auf landwirtschaftlichen Rand- und Restflächen

- Baugebietsrückzonung

- Privatwaldzusammenlegung, Erwerb von Privatwaldparzellen durch die Öffentlichkeit zur Gewährleistung einer geregelten Bewirtschaftung

- Entflechtung der Erholungsaktivitäten durch Beschilderung verschiedener Wegarten gemäß grenzüberschreitendem Konzept

- Erlaß eines Reglementes für den Modellflugsport

- Teilweise Aufhebung des Velofahrverbotes auf Flurwegen, Erlaß von Fahrverboten und Bau von Sammelparkplätzen

- Ausstattung geeigneter Flächen für Picknick, Übernahme von Spielwiesen in das Eigentum der Gemeinde

- Zonenmäßige Festlegung des Intensiverholungsgebietes

- Schutzzonenausscheidung für die wertvolle Landschaftssubstanz

- Maßnahmen zur Gestaltung innerörtlicher Grünzonen
- Regelung des Lehmabbaus Ziegelei durch spezielle Zonenvorschriften

- Intensive Einpflanzung des Baukomplexes Gymnasium mit Quartierplan

- Festlegung der zulässigen Nutzungen und der erforderlichen Pflegemaßnahmen in den Naturund Landschaftsschutzgebieten über Zonenvorschriften

- Erstellen eines Grünordnungsplanes als Grund. lage für alle notwendigen Gestaltungs- und Landschaftspflegemaßnahmen im Bereich Bir. sig-Bernhardsberg.

\section{Gemeindeerlaß}

Die eben zitierten Postulate wurden - soweit sie mittels Raumplanungsmaßnahmen realisierbar sind - im Erlaß der Gemeinde zur Landschaftsordnung verwirklicht. Dieser umfaßt unter dem Titel «Landschafts-Zonenvorschriften» einen Zonenplan Maßstab 1:5000, die kantonalen Zonenreglement-Normalien Landschaft und Ergänzungsbestimmungen der Gemeinde hiezu. Die letzteren und die Normalien bilden zusammen das Reglement zum Planerlaß, welches insbesondere die Nutzungsausübung in den ausgeschiedenen Zonen regelt.

Der Zonenplan Landschaft enthält folgende Elemente:

\section{Nutzungszonen:}

Zonen für Wochenendhäuser, öffentliche Zonen für Familiengärten, Gartenbad, Freizeit, Tennis, Sport und Leichtathletik, ferner Grünzonen, Spezialzonen für Intensiverholung, Gärtnereien und für Ausbeutung.

Schutzzonen und -objekte:

Naturschutz-, Landschaftsschutz- und Aussichtsschutzzonen, Naturschutz-Einzelobjekte, Kleinbiotope, botanische und kulturhistorische Objekte.

Zur Orientierung (nicht Gegenstand der Beschlußfassung):

Land- und Forstwirtschaftsgebiet, Gewässer, sowie bereits rechtskräftige Zonen und geschützte Elemente.

Die Landschaftszonenvorschriften fanden in der Schlußabstimmung an der Gemeindeversammlung vom 26. April 1979 einstimmige Annahme. 


\section{Schlußbetrachtung}

Der Planungserfolg ist wesentlich auf das Konto des Gemeinderates und insbesondere auf dessen umsichtigen Bauchef $\mathrm{zu}$ buchen. Die kritische Phase umfassender Öffentlichkeitsinformation wurde von ihm sehr minutiös und umfassend angelegt. Es fanden echte Dialoge mit der betroffenen Bevölkerung statt, wobei sich der Gemeinderat als flexibler Partner erwies, der sich auf berechtigte Anliegen einzulassen wußte. Von Bedeutung war sicher auch, daß schon während der Planungsarbeit hinreichende Kontakte zu den betroffenen Kreisen gepflegt wurden. Ein derartiger Dialog kann nur erfolgreich sein, wenn vorgängig nicht allein die Probleme durch Planer und Spezialkommission genügend tief ausgelotet und faßlich dargestellt werden, sondern darüber hinaus ein durchdachtes Konzept zu ihrer Lösung auf den Tisch gelegt wird, über welches eine fruchtbare Diskussion erst möglich wird.

Es bleibt zu hoffen, daß möglichst bald auch die anderen Gemeinden zum Schutze des wertvollen Gutes «Landschaft» und damit zu ihrem eigenen Wohl vom Instrument der Landschaftsplanung auf Gemeindeebene Gebrauch machen werden. Das Beispiel Oberwil möge sie dazu ermuntern.

\section{Literatur:}

Planergemeinschaft Regionalplanung LaufentalThierstein-Dorneck: Technischer Bericht zum regionalen Richtplan Landschaft, 10. Oktober 1978, Verfasser: M. BUUTLER, J. WIEDE

Regierungsrat des Kantons Basel-Landschaft:

Regionalplan Landschaft, Vorlage an den Landrat, 3. Mai 1977. C: Regionalplanungsstelle beider Basel, Liestal

Regionalkonferenz der Regierungen der Nordwestschweiz: Generelles Landschaftskonzept Nord- westschweiz. Verfasser: M. BÜTLER und J. WIEDE in Zusammenarbeit mit DR. K. EWALD und den kantonalen Amtsstellen für Raumplanung, Liestal, 1978

\section{Résumé}

L'intérêt porté à l'aménagement du territoire se concentrait autrefois presque uniquement sur les questions de l'urbanisation et de transports. Mais aujourd'hui, le public a pris conscience des nombreux problèmes de toute nature que pose la préservation même du paysage non bâti.

La protection du paysage consiste à restreindre l'usage du sol à l'exterieur des zones à bâtir dans le dessein de le subordonner à la sauvegarde. Dans la région nord-ouest de la Suisse, un accord conclu entre les cantons d'Argovie, de Bâle-Campagne, Bâle-Ville, Soleure et Berne a réuni dans une conception uniforme dite «Landschaftskonzept Nordwestschweiz» les catégories de sauvegarde définies par les termes de «Protection de la nature», «Protection du paysage» et "Respect du paysage».

Oberwil, commune de la banlieue bâloise située dans le vallon de Leimen, a récemment fait approuver par l'assemblée communale un plan et un règlement d'aménagement du paysage local. Ce décret complète les dispositions déjà en viguer pour la zone à bâtir. Il règle les limites dans lesquelles il sera permis d'utiliser le reste du territoire communal.

Les autorités sont convaincues que le plan adopté constituera un instrument efficace permettant de délester sensiblement la commune de la pression qu'exercent sur elle les incessantes demandes tendant à l'établissement d'installations de loisirs et de récréation. Elles espérent ainsi soustraire à une utilisation déraisonnable les éléments du paysage dont l'aspect et l'écologie méritent sauvegarde, et assurer à l'agriculture et à la sylviculture les bases nécessaires à leur existence. 